\title{
Global fixed points for centralizers and Morita's Theorem
}

\author{
JOHN FRANKS \\ MichaEL HANDEL
}

\begin{abstract}
We prove a global fixed point theorem for the centralizer of a homeomorphism of the two-dimensional disk $D$ that has attractor-repeller dynamics on the boundary with at least two attractors and two repellers. As one application we give an elementary proof of Morita's Theorem, that the mapping class group of a closed surface $S$ of genus $g$ does not lift to the group of $C^{2}$ diffeomorphisms of $S$ and we improve the lower bound for $g$ from 5 to 3 .
\end{abstract}

37E30, 57M60, 37C25

\section{Introduction}

In this article we are concerned with the properties of groups of homeomorphisms or diffeomorphisms of surfaces. We assume throughout that $S$ is a surface of finite negative Euler characteristic, without boundary but perhaps with punctures. We denote the group of orientation preserving homeomorphisms of $S$ and the group of orientation preserving $C^{1}$ diffeomorphisms of $S$ by $\operatorname{Homeo}(S)$ and $\operatorname{Diff}(S)$ respectively and we denote the subgroups consisting of elements that are isotopic to the identity by $\mathrm{Homeo}_{0}(S)$ and $\operatorname{Diff}_{0}(S)$ respectively.

An important tool in the study of subgroups of these groups is the existence of a global fixed point. A global fixed point for a subgroup $\mathcal{G}$ of $\operatorname{Homeo}(S)$ is a point $x \in S$ that is fixed by each element of $\mathcal{G}$. The set of global fixed points for $\mathcal{G}$ is denoted $\operatorname{Fix}(\mathcal{G})$. When $G$ is a subgroup of $\operatorname{Diff}(S)$ and $\operatorname{Fix}(\mathcal{G})$ is nonempty, the assignment $g \mapsto D g_{x}$ (the derivative of $g$ at $x \in \operatorname{Fix}(\mathcal{G})$ ), gives a representation of $\mathcal{G}$ in $G l(2, \mathbb{R})$. This representation can be very useful for understanding $\mathcal{G}$; for example in our paper [8] this representation was used to prove that many lattices, including $S L(3, \mathbb{Z})$, are not isomorphic to a subgroup of the group of measure preserving diffeomorphisms of a surface.

Unfortunately, there are no general techniques for finding a global fixed point for a subgroup of Homeo $(S)$. In particular there are no analogues of the standard tools of algebraic topology for finding fixed points of a single map. In the case of surfaces 
there is a literature concerning the existence of global fixed points, but it is largely limited to abelian groups (see, eg Bonatti [1; 2], Franks, Handel and Parwani [10; 9] and Handel [11]).

The main objective of this article is to provide a technique which, in many cases, allows us to find a global fixed point for the centralizer of $f \in \operatorname{Homeo}(S)$. We denote the centralizer of $f$ by $\operatorname{Cent}(f)$ and observe that it can be a very large group and far from abelian, for example when $\operatorname{Fix}(f)$ has interior.

As one application of this result we address the "lifting problem" for the mapping class group (see Section 6 of Farb [5]). Using our result on global fixed points and the representation in $G l(2, \mathbb{R})$ mentioned above, we give an elementary proof of an important theorem of Morita about the nonexistence of liftings of the full mapping class group to $\operatorname{Diff}^{2}(S)$ and improve the lower bound on the genus of $S$ required for the result (see Theorem 1.5 below).

The closed two-dimensional disk is denoted $D$. The universal cover $\widetilde{S}$ of $S$ is naturally identified with int $D$ and the compactification of $\widetilde{S}$ by the circle at infinity $S_{\infty}$ is naturally identified with $D$. For this reason, our main result concerns global fixed points for group actions on $D$.

Theorem 1.1 Let $\mathcal{G}$ be a subgroup of $\operatorname{Homeo}(D)$ and let $f$ be an element of the center of $\mathcal{G}$. Suppose $K:=\operatorname{Fix}(f) \cap \partial D$ consists of a finite set with more than two elements each of which is either an attracting or repelling fixed point for $f: D \rightarrow D$. Let $\mathcal{G}_{0} \subset \mathcal{G}$ denote the finite index subgroup whose elements pointwise fix $K$. Then $\operatorname{Fix}\left(\mathcal{G}_{0}\right) \cap \operatorname{int}(D)$ is nonempty.

The hypothesis of this theorem has both an algebraic part, namely that $\mathcal{G}_{0} \subset \operatorname{Cent}(f)$, and a dynamical part, namely that $\operatorname{Fix}(f) \cap \partial D$ consists of points which are attractors or repellers. The latter implies that $\operatorname{Fix}(f) \cap$ int $D \neq \varnothing$ as a consequence of the Lefschetz index theorem. The former is used to relate the dynamics of elements of $\mathcal{G}_{0}$ to $f$ and hence to each other.

The fixed point set $\operatorname{Fix}(f)$ of $f \in \operatorname{Homeo}(S)$ is partitioned into Nielsen classes. Two elements $x, y \in \operatorname{Fix}(f)$ belong to the same Nielsen class if there is a lift $\tilde{f}: \widetilde{S} \rightarrow \tilde{S}$ of $f: S \rightarrow S$ and lifts $\tilde{x}, \tilde{y} \in \operatorname{Fix}(\tilde{f})$ of $x$ and $y$. Equivalently, every lift of $f$ that fixes a lift of $x$ also fixes a lift of $y$. If $f$ is isotopic to $g$ and $\tilde{f}$ is a lift of $f$ then the isotopy between $f$ and $g$ lifts to an isotopy between $\tilde{f}$ and a lift $\tilde{g}$ of $g$. We say that $\tilde{f}$ and $\tilde{g}$ are paired. Pairing defines a bijection between lifts of $f$ and lifts of $g$ and we say that the $f$-Nielsen class of $x \in \operatorname{Fix}(f)$ is paired with the $g$-Nielsen class of $z \in \operatorname{Fix}(g)$ if there are paired lifts $\tilde{f}$ and $\tilde{g}$ of $f$ and $g$ and there are lifts $\tilde{x} \in \operatorname{Fix}(\tilde{f})$ of $x$ and $\tilde{z} \in \operatorname{Fix}(\widetilde{g})$ of $z$. 
By a pseudo-Anosov homeomorphism of a punctured surface we mean the restriction of a homeomorphism of the unpunctured surface that is pseudo-Anosov relative to the set of punctures.

Theorem 1.2 Suppose that $\alpha \in \operatorname{Homeo}(S)$ is pseudo-Anosov and that $f \in \operatorname{Homeo}(S)$ is isotopic to $\alpha$. Let $\mathcal{H}_{0}=\operatorname{Cent}(f) \cap \operatorname{Homeo}_{0}(S)$. Then $\operatorname{Fix}\left(\mathcal{H}_{0}\right)$ is infinite. More precisely, for each $n \geq 0$ and each $y \in \operatorname{Fix}\left(\alpha^{n}\right)$ there exists $x \in \operatorname{Fix}\left(\mathcal{H}_{0}\right) \cap \operatorname{Fix}\left(f^{n}\right)$ such that the Nielsen class of $f^{n}$ determined by $x$ is paired with the Nielsen class of $\alpha^{n}$ determined by $y$.

In the special case that $f=\alpha$, the group $\mathcal{H}_{0}$ is trivial (see, eg Travaux de Thurston sur les surfaces [6]) and Theorem 1.2 is obvious. Thus Theorem 1.2 fits into the general scheme of results in surface dynamics in which an important property of pseudo-Anosov maps is extended to all elements of its isotopy class.

Remark 1.3 Suppose that $f^{\prime}: T^{2} \rightarrow T^{2}$ is isotopic to a linear Anosov homeomorphism $\alpha^{\prime}: T^{2} \rightarrow T^{2}$ and that $e \in \operatorname{Fix}(\alpha)$ is the image of $(0,0) \in \mathbb{R}^{2}$ under the usual covering map. Assume $e \in \operatorname{Fix}\left(f^{\prime}\right)$. Let $S=T^{2} \backslash\{e\}$, let $f=\left.f^{\prime}\right|_{S}$ and let $\alpha=\left.\alpha^{\prime}\right|_{S}$. Then $\alpha$ is pseudo-Anosov and we may apply Theorem 1.2. The conclusions are exactly as given in the theorem but are applied to the subgroup $\mathcal{H}_{0}^{\prime}$ of $\operatorname{Cent}\left(f^{\prime}\right)$ that are isotopic to the identity relative to $e$.

Our next result is the analogue of Theorem 1.2 for reducible isotopy classes with a pseudo-Anosov component. It is a corollary of, and the original motivation for, Theorem 1.1, since it provides the tool we use to prove Morita's theorem.

Theorem 1.4 Suppose that $f \in \operatorname{Homeo}(S)$, that $S_{0} \subset S$ is an incompressible subsurface and that $f$ is isotopic to $\alpha \in \operatorname{Homeo}(S)$ where $\alpha\left(S_{0}\right)=S_{0}$ and $\left.\alpha\right|_{S_{0}}$ is pseudoAnosov. Let $\mathcal{H}_{0}$ be the subgroup of $\operatorname{Cent}(f)$ consisting of elements that are isotopic to a homeomorphism that pointwise fixes $S_{0}$. Then $\operatorname{Fix}\left(\mathcal{H}_{0}\right)$ is infinite. More precisely, for each $n \geq 0$ and each $y \in \operatorname{Fix}\left(\alpha^{n}\right) \cap \operatorname{int}\left(S_{0}\right)$ there exists $x \in \operatorname{Fix}\left(\mathcal{H}_{0}\right) \cap \operatorname{Fix}\left(f^{n}\right)$ such that the Nielsen class of $f^{n}$ determined by $x$ is paired with the Nielsen class of $\alpha^{n}$ determined by $y$.

The mapping class group $\mathrm{MCG}(S)$ of a closed surface $S$ is the group of isotopy classes of orientation preserving homeomorphisms of $S$. There is a natural homomorphism $\operatorname{Homeo}(S) \rightarrow \operatorname{MCG}(S)$ that sends $h \in \operatorname{Homeo}(S)$ to its isotopy class $[h] \in \operatorname{MCG}(S)$. A lift of a subgroup $\Gamma$ of $\operatorname{MCG}(S)$ is a homomorphism $\Gamma \rightarrow \operatorname{Homeo}(S)$ so that the composition

$$
\Gamma \rightarrow \operatorname{Homeo}(S) \rightarrow \operatorname{MCG}(S)
$$


is the inclusion. Every free abelian subgroup (see Section 6.3 of Farb [5]) and every finite subgroup (see Kerckhoff [14]) of $\operatorname{MCG}(S)$ has a lift to $\operatorname{Diff}(S)$. Morita [18; 19] proved that $\operatorname{MCG}(S)$ does not lift to $\operatorname{Diff}^{2}(S)$ for genus $(S) \geq 5$ and Markovich [16] proved that $\operatorname{MCG}(S)$ does not lift to $\operatorname{Homeo}(S)$ for $\operatorname{genus}(S) \geq 6$.

Using Theorem 1.4 and the Thurston stability theorem we give an elementary proof of Morita's theorem and improve the lower bound on the genus.

Theorem 1.5 Suppose $S$ is a closed surface. Then $\operatorname{MCG}(S)$ does not lift to $\operatorname{Diff}(S)$ for $\operatorname{genus}(S) \geq 3$.

This is actually a special case of a more general result in which we consider homomorphisms $\mathcal{L}: \Gamma \rightarrow \operatorname{Diff}(S)$ that are not necessarily lifts.

Suppose that a closed surface $S=S_{1} \cup S_{2}$ where $S_{1}$ and $S_{2}$ are incompressible subsurfaces with disjoint interiors. Recall that the relative mapping class group $\operatorname{MCG}\left(S_{i}, \partial S_{i}\right)$ is the set of isotopy classes rel $\partial S_{i}$ of homeomorphisms $h: S_{i} \rightarrow S_{i}$ that are the identity on $\partial S_{i}$. Each such $h$ extends by the identity to a homeomorphism of $S$. This induces a homomorphism $\Phi: \operatorname{MCG}\left(S_{i}, \partial S_{i}\right) \rightarrow \operatorname{MCG}(S)$. If $S$ is given a hyperbolic structure with $\partial S_{i}$ a union of geodesics, it is straightforward to see that any $f \in \operatorname{Homeo}_{0}(S)$ is actually homotopic to the identity along geodesics, ie with $f_{t}(x)$ on the geodesic from $x$ to $f(x)$ determined by the isotopy. From this it follows that if $f$ is the identity on the complement of $S_{i}$, then we may choose a homotopy from $f$ to the identity with the same property, and hence an isotopy of $\left.f\right|_{S_{i}}$ to the identity rel $\partial S_{i}$. Therefore the homomorphism $\Phi$ is injective and we use it to identify $\operatorname{MCG}\left(S_{i}, \partial S_{i}\right)$ with a subgroup of $\operatorname{MCG}(S)$.

Theorem 1.6 Assume notation as above and that $\Gamma=\left\langle\Gamma_{1}, \mu\right\rangle$ where

- $\Gamma_{1}$ is a nontrivial finitely generated subgroup of $\operatorname{MCG}\left(S_{1}, \partial S_{1}\right)$ such that $H^{1}\left(\Gamma_{1}, \mathbb{R}\right)=\{0\}$,

- $\mu \in \operatorname{MCG}\left(S_{2}, \partial S_{2}\right)$.

Then there does not exist a faithful homomorphism $\mathcal{L}: \Gamma \rightarrow \operatorname{Diff}(S)$ such that

(1) $\left.\left[\mathcal{L}\left(\Gamma_{1}\right)\right] \subset \operatorname{MCG}\left(S_{1}, \partial S_{1}\right)\right)$,

(2) $[\mathcal{L}(\mu)] \in \operatorname{MCG}\left(S_{2}, \partial S_{2}\right)$ is represented by $\alpha: S \rightarrow S$ where $\alpha\left(S_{2}\right)=S_{2}$ and $\alpha \mid S_{2}$ is pseudo-Anosov. 
Theorem 5.1 of Korkmaz [15] asserts that if $S$ is a compact surface of genus at least three with $\partial S$ either empty or with one component, then $H_{1}(\operatorname{MCG}(S, \partial S), \mathbb{Z})$ and $H_{1}(\operatorname{MCG}(S), \mathbb{Z})$ are both trivial. Moreover, in the case of genus 2 , both of these groups are isomorphic to $\mathbb{Z} / 10 \mathbb{Z}$. Hence whenever the genus is at least two both $H^{1}(\operatorname{MCG}(S), \mathbb{R})$ and $H^{1}(\operatorname{MCG}(S, \partial S), \mathbb{R})$ are trivial. Theorem 1.5 follows from these facts and Theorem 1.6. It is conjectured that when the genus is at least two $H^{1}(\Gamma, \mathbb{R})$ is trivial for all finite index subgroups $\Gamma$ of $\operatorname{MCG}(S)$ or $\operatorname{MCG}(S, \partial S)$. If that conjecture is verified for $\operatorname{MCG}(S, \partial S)$ then Theorem 1.6 will imply that no finite index subgroup of $\operatorname{MCG}(S)$ lifts to $\operatorname{Diff}(S)$, which is known for genus at least 5 because Morita's original proof applies to all finite index subgroups of $\operatorname{MCG}(S)$.

Acknowledgements We thank Benson Farb for several very helpful conversations. The first author was supported in part by NSF grant DMS-055463. The second author was supported in part by NSF grant DMS-0103435.

\section{A global fixed point theorem}

We begin with generalities about attracting fixed points.

Suppose that $f: X \rightarrow X$ is a homeomorphism of a locally compact metric space $X$ and that $x_{0} \in \operatorname{Fix}(f)$. We say that $x_{0}$ is an attracting fixed point for $f$ if there is a compact neighborhood $W$ of $x_{0}$ such that the $f^{n}(W) \rightarrow\left\{x_{0}\right\}$ in the Hausdorff topology; ie for every neighborhood $N$ of $x_{0}$, we have $f^{n}(W) \subset N$ for all sufficiently large $n$. The basin of attraction of $x_{0}$ with respect to $f$ is defined to be $\{x \in X$ : $\left.\lim _{n \rightarrow \infty} f^{n}(x)=x_{0}\right\}$. Note that the basin of attraction of $x_{0}$ is $f$-invariant and contains $W$.

Remark 2.1 If $W$ is a compact neighborhood of $x_{0}$ such that $f(W) \subset W$ then $f^{n}(W) \rightarrow\left\{x_{0}\right\}$ in the Hausdorff topology if and only if $\bigcap_{n=1}^{\infty} f^{n}(W)=\left\{x_{0}\right\}$. Thus by item (2) of Lemma 2.2 below, $x_{0}$ is an attracting fixed point if and only if it has a compact neighborhood $W$ such that $f(W) \subset W$ and $\bigcap_{n=1}^{\infty} f^{n}(W)=\left\{x_{0}\right\}$.

If $x$ is an attracting fixed point for $f^{-1}$ then it is a repelling fixed point for $f$.

Lemma 2.2 Let $f: X \rightarrow X$ be a homeomorphism of a locally compact metric space with an attracting fixed point $x_{0} \in X$ and basin of attraction $U$.

(1) For any compact neighborhood $W_{0} \subset U$ of $x_{0}$,

$$
\bigcup_{i=0}^{\infty} f^{-i}\left(W_{0}\right)=\bigcup_{i=0}^{\infty} f^{-i}\left(\text { int } W_{0}\right)=U \text {. }
$$


(2) There exist arbitrarily small compact neighborhoods $V$ of $x$ such that $f(V) \subset V$.

(3) For any compact set $A \subset U, \lim _{n \rightarrow \infty} f^{n}(A)=\left\{x_{0}\right\}$ in the Hausdorff topology.

(4) If $x_{0}$ is also an attracting fixed point for a homeomorphism $g: X \rightarrow X$ that commutes with $f$ then $U$ is the basin of attraction of $x$ with respect to $g$.

Proof Let $W$ be a compact neighborhood of $x_{0}$ as in the definition of attracting fixed point.

Suppose that $W_{0} \subset U$ is a compact neighborhood of $x_{0}$. Since $U$ is $f^{-1}$-invariant, $\bigcup_{i=0}^{\infty} f^{-i}\left(W_{0}\right) \subset U$. Conversely, if $x \in U$ then $f^{i}(x) \in$ int $W_{0}$ for all sufficiently large $i$. This proves that $U \subset \bigcup_{i=0}^{\infty} f^{-i}$ (int $\left.W_{0}\right) \subset \bigcup_{i=0}^{\infty} f^{-i}\left(W_{0}\right) \subset U$ which proves (1).

For (2), choose $q>0$ such that $f^{q}(W) \subset \operatorname{int}(W)$ and define $V_{1}=\bigcup_{k=0}^{q} f^{k}(W) \subset U$. Then $f\left(V_{1}\right) \subset V_{1}$. Given a neighborhood $N$ of $x$ choose $l \geq 0$ so that $f^{l}\left(V_{1}\right) \subset N$ and let $V=f^{l}\left(V_{1}\right)$. Then $V \subset N$ and $f(V) \subset V$.

By (1) and (2) there is a compact neighborhood $V \subset W$ of $x$ such that $f(V) \subset V$ and such that $U=\bigcup_{i=0}^{\infty} f^{-i}$ (int $V$ ). If $A$ is a compact subset of $U$ then $A \subset f^{-m}$ (int $V$ ) for some $m>0$. Thus $f^{i}(A) \subset V$ for all $i \geq m$ and we conclude $\lim _{n \rightarrow \infty} f^{n}(A) \subset$ $\bigcap_{i=0}^{\infty} f^{i}(V) \subset \bigcap_{i=0}^{\infty} f^{i}(W)=\left\{x_{0}\right\}$. This proves (3).

Suppose now that $g$ is as in (4) and that $U^{\prime}$ is the basin of attraction of $x_{0}$ with respect to $g$. For any compact neighborhood $N \subset U \cap U^{\prime}$ of $x_{0}, U=\bigcup_{i=0}^{\infty} f^{-i}$ (int $N$ ) and $U^{\prime}=$ $\bigcup_{j=0}^{\infty} g^{-j}$ (int $\left.N\right)$ by (1). For all $i \geq 0$ there exists $j \geq 0$ so that $g^{j}(N) \subset f^{i}($ int $N)$. Thus $g^{j}\left(f^{-i}(N)\right)=f^{-i}\left(g^{j}(N)\right) \subset \operatorname{int} N$ or equivalently $f^{-i}(N) \subset g^{-j}($ int $N)$. This proves that $U \subset U^{\prime}$. The reverse inclusion follows by symmetry.

Lemma 2.3 Let $f: X \rightarrow X$ be a homeomorphism of a locally compact metric space and let $x_{0} \in X$ be an attracting fixed point for $f$. If $g: X \rightarrow X$ is a homeomorphism that commutes with $f$ and fixes $x_{0}$ then there exists $m>0$ such that $x_{0}$ is an attracting fixed point for $h=f^{m} g$.

Proof Let $U$ be the basin of attraction for $x_{0}$ with respect to $f$. Then $g(U)$ is the basin of attraction for $x_{0}$ with respect to $g f g^{-1}=f$ which implies that $g(U)=U$. By part (2) of Lemma 2.2 there is a compact neighborhood $V$ of $x_{0}$ in $U$ such that $f(V) \subset V$. By part (3) of the same lemma there is $m>0$ such that $f^{m}(g(V)) \subset f(V)$.

Define $h=f^{m} g$. Thus $h(V) \subset f(V) \subset V$. Applying $h^{n-1}$ we conclude $h^{n}(V) \subset$ $f h^{n-1}(V)$ for all $n$. Hence

$$
f^{-1} h^{n}(V) \subset h^{n-1}(V)
$$


for all $n$.

Let $\Lambda$ be the nonempty compact set $\bigcap_{n \geq 0} h^{n}(V)$. Then $f(\Lambda) \subset \Lambda$ because $f(V) \subset V$. Also, the displayed inclusion above implies $f^{-1}(\Lambda) \subset \Lambda$. We conclude that $f(\Lambda)=\Lambda$ so by part (3) of Lemma 2.2 the only possibility is that $\Lambda=\left\{x_{0}\right\}$. By Remark 2.1, this proves that $x_{0}$ is an attracting fixed point for $h$.

We now turn to the proof of our main result.

Proof of Theorem 1.1 The points of $K$ are attractors or repellers for $f$ and hence $a$ fortiori attractors or repellers for $\left.f\right|_{\partial D}$. Hence there must be an equal number of attractors and repellers which alternate on $\partial D$. We will denote the attractors $\left\{p_{1}, \ldots, p_{k}\right\}$ and the repellers $\left\{q_{1}, \ldots, q_{k}\right\}$ in their circular order.

For simplicity it is useful to consider the two sphere $S^{2}$ obtained by doubling $D$ along its boundary. There is a natural extension of $f$ to $S^{2}$ which we will also denote by $f$. Then $\left\{p_{1}, \ldots, p_{k}\right\}$ are attracting fixed points of $f: S^{2} \rightarrow S^{2}$ and $\left\{q_{1}, \ldots, q_{k}\right\}$ are repelling fixed points. Each element of $\mathcal{G}_{0}$ also extends in a natural way to $S^{2}$ and abusing notation we will denote this group by $\mathcal{G}_{0}$.

Let $\mathcal{H}$ be the set of elements of $\left\langle\mathcal{G}_{0}, f\right\rangle$ for which each $p_{i}$ is an attractor and each $q_{i}$ is a repeller. Then $f \in \mathcal{H}$ and by Lemma 2.3, for all $g \in \mathcal{G}_{0}$ there exists $m>0$ such that $f^{m} g \in \mathcal{H}$. Our goal is to find $y \in S^{2}$, not in the set $\left\{p_{1}, \ldots, p_{k}\right\} \cup\left\{q_{1}, \ldots, q_{k}\right\}$, such that $y \in \operatorname{Fix}(h)$ for each $h \in \mathcal{H}$. We then observe that $y \in \operatorname{Fix}(f) \cap \operatorname{Fix}\left(f^{m} g\right) \subset \operatorname{Fix}(g)$ for all $g \in \mathcal{G}_{0}$ which will complete the proof.

Remark 2.4 An easy index argument shows that for each $h \in \mathcal{H}$ there is at least one element of Fix $(h)$ that has negative index and so is neither a source nor a sink. The challenge here is to find a single point that works for all $h$. The point we find in $\operatorname{Fix}(\mathcal{H})$ will be shown to be neither a source nor a sink for any element $h \in \mathcal{H}$, but we do not know about its index.

Lemma 2.2 (4) and the fact that each $h \in \mathcal{H}$ commutes with $f$ imply that the basin of attraction $U$ for $p_{1}$ with respect to $h \in \mathcal{H}$ is independent of $h$. By Lemma 2.2 (1), $U$ can be written as an increasing union of open disks and so is connected and simply connected. The fact that there is another attractor $p_{2}$ implies that the frontier of $U$ is not a single point.

We will be interested in two compactifications of $U$. The first is $\bar{U}$, the closure of $U$ in $S^{2}$ and the second is $\widehat{U}$, the prime end compactification. The set $\Theta=\hat{U} \backslash U$ of prime ends is topologically a circle. Each homeomorphism $\left.h\right|_{U}: U \rightarrow U$ extends to a homeomorphism $\hat{h}: \widehat{U} \rightarrow \widehat{U}$. Moreover, the following holds: 
(*) For each continuous arc $\gamma:[0,1] \rightarrow \bar{U}$ with $\gamma([0,1)) \subset U$ and $\gamma(1)$ in the frontier of $U$ there is a continuous arc $\hat{\gamma}:[0,1] \rightarrow \widehat{U}$ with $\hat{\gamma}(t)=\gamma(t)$ for $t \in[0,1)$. The point $\gamma(1)$ is called an accessible point of the frontier of $U$ and $\hat{\gamma}(1)$ is a prime end corresponding to it (there may be more than one prime end corresponding to an accessible point).

These properties go back to Caratheodory. An excellent modern exposition can be found in Mather's paper [17]. In particular see Section 17 of [17] for a discussion of accessible points.

Let $C$ denote the arc in $\partial D$ joining $p_{1}$ and $q_{1}$ and not containing any other points of Fix $(f)$. Then $C \subset \bar{U}$ and all but the endpoint $q_{1}$ of $C$ lies in $U$. By $(*)$, the half open arc $C \cap U$ converges to a prime end $\hat{w} \in \Theta$ that is fixed by each $\hat{h}$ since $C$ is $h$-invariant for each $h$.

Our first objective is to show that $\widehat{w}$ is a repelling fixed point for each $\left.\widehat{h}\right|_{\Theta}$ or equivalently an attracting fixed point for each $\left.\hat{h}^{-1}\right|_{\Theta}$. For this, there is no loss in replacing $h$ by an iterate so by applying Lemma 2.2 (3) we may choose a disk neighborhood $D_{1}$ of $q_{1}$ in the basin of attraction of $q_{1}$ with respect to $h^{-1}$ such that $h^{-1}\left(D_{1}\right) \subset \operatorname{int}\left(D_{1}\right)$. Let $\gamma_{0}$ denote the component of $\partial D_{1} \cap U$ which intersects $C$, and let $\gamma_{i}=h^{-i}\left(\gamma_{0}\right)$. Note that each $\gamma_{i}$ intersects $C$ and is the interior of a closed path $\bar{\gamma}_{i} \subset \bar{U}$ and so by (*) is the interior of a closed path $\hat{\gamma}_{i} \subset \hat{U}$. The $\gamma_{i}$ 's separate $U$ into two complementary components $V_{i}$ and $W_{i}$ with $W_{i+1} \subset W_{i}$ and $\bigcap_{i=1}^{\infty} W_{i}=\varnothing$ (where the last property follows from Remark 2.1). By Corollary 4 of [17], the $\hat{\gamma}_{i}$ 's converge to a single prime end, which must be $\hat{w}$ since each $\hat{\gamma}_{i}$ intersects $C$. The $\hat{h}^{-1}$-orbit of the endpoints of $\hat{\gamma}_{i}$ converge to $\widehat{w}$. This proves that $\widehat{w}$ is an attractor for $\left.\hat{h}^{-1}\right|_{\Theta}$.

The next step is to find a prime end $\hat{y}$ that is fixed by each $\hat{h}$ and that does not come from $\partial D$. Since each $\hat{h}$ commutes with $\hat{f}$, Lemma 2.2 (4) implies that the interval of attraction of $\hat{w}$ with respect to $\hat{h}^{-1}$ is independent of $h$. Let $\hat{y}$ be one endpoint of this interval of attraction and let $J$ be the interval in $\Theta$ with endpoints $\widehat{w}$ and $\hat{y}$ which lies in the basin of $\widehat{w}$. The only fixed points of $\left.\hat{h}\right|_{J}$ are the endpoints and $\widehat{w}$ is a repeller while $\hat{y}$ is an attractor.

Now we show how to extract a (not necessarily unique) point $y \in \bar{U}$ from $\hat{y}$ that is fixed by each $h$. By Corollary 11 and Theorem 13 of [17] there is a sequence of disjoint closed arcs $\bar{\alpha}_{i} \subset \bar{U}$ with interior $\alpha_{i} \subset U$ and endpoints in the frontier of $U$ (in $S^{2}$ ) and there exists $y$ in the frontier of $U$ such that

- $\bar{\alpha}_{i} \rightarrow y$ (in the Hausdorff topology on $S^{2}$ ),

- there is a component $Z_{i}$ of $\left(U \backslash \alpha_{i}\right)$ such that $Z_{i+1} \subset Z_{i}$ and $\bigcap_{i=1}^{\infty} Z_{i}=\varnothing$, 
- $\alpha_{i}$ extends to a closed arc $\widehat{\alpha}_{i}$ in $\widehat{U}$ such that $\widehat{\alpha}_{i}$ converges to $\hat{y}$ (in the Hausdorff topology on $\widehat{U}$ ).

Clearly if we show $h\left(\alpha_{i}\right) \cap \alpha_{i} \neq \varnothing$ then it follows that $y \in \operatorname{Fix}(h)$. One of the endpoints of $\widehat{\alpha}_{i}$, call it $\hat{x}_{i}$, lies in $J$. Since $\hat{h}$ has no fixed points in the interior of $J$ it follows that

$$
\lim _{n \rightarrow \infty} \hat{h}^{n}\left(\hat{x}_{i}\right)=\hat{y} \quad \text { and } \quad \lim _{n \rightarrow-\infty} \hat{h}^{n}\left(\hat{x}_{i}\right)=\widehat{w} .
$$

On the other hand each $z \in \alpha_{i}$ lies in $U$, the basin of attraction of $p_{1}$, so

$$
\lim _{n \rightarrow \infty} h^{n}(z)=p_{1} .
$$

Since $\hat{\alpha}_{i}$ separates $\hat{y}$ and $p_{1}$ in $\widehat{U}$ we conclude that $h^{n}\left(\alpha_{i}\right) \cap \alpha_{i} \neq \varnothing$ for large $n$ (and hence also $\left.\alpha_{i} \cap h^{-n}\left(\alpha_{i}\right) \neq \varnothing\right)$. But this implies $h^{k}\left(\alpha_{i}\right) \cap \alpha_{i} \neq \varnothing$ for all $k \in \mathbb{Z}$ because $h^{k}\left(\alpha_{i}\right) \cap \alpha_{i}=\varnothing$ and $k \neq 0$ would imply that for all $n>0$ either $h^{n k}\left(\alpha_{i}\right) \subset Z_{i}$ or $h^{-n k}\left(\alpha_{i}\right) \subset Z_{i}$ which is a contradiction. Hence the point $y$ is fixed by each $h$.

Moreover, for any $h \in \mathcal{H}$ we have $h^{k}\left(\alpha_{i}\right) \cap \alpha_{i} \neq \varnothing$ for all $k \in \mathbb{Z}$. This implies $y$ is not an attractor for either $h$ or $h^{-1}$ since otherwise, choosing $A=\bar{\alpha}_{i}$ for some large $i$, we would contradict Lemma 2.2 (3). We conclude $y$ is not contained in $\left\{p_{1}, \ldots, p_{k}\right\} \cup\left\{q_{1}, \ldots, q_{k}\right\}$.

\section{Applications}

Proof of Theorem 1.2 and of Theorem 1.4 We assume the notation of Theorem 1.4 and allow the possibility that $S_{0}=S$.

We use the standard setup for discussing Nielsen classes in surfaces; further details can be found, for example, in Section 3 of Handel [12]. The universal cover $\widetilde{S}$ of $S$ is topologically the interior of a disk and is compactified to a closed disk $D$ by the "circle at infinity' $S_{\infty}$. Every lift $\tilde{g}: \widetilde{S} \rightarrow \widetilde{S}$ of every homeomorphism $g: S \rightarrow S$ extends to a homeomorphism $\hat{g}: D \rightarrow D$. If an isotopy between $g_{1}$ and $g_{2}$ is lifted to an isotopy between lifts $\widetilde{g}_{1}$ and $\tilde{g}_{2}$ then $\left.\hat{g}_{1}\right|_{S_{\infty}}=\left.\hat{g}_{2}\right|_{S_{\infty}}$.

Choose a component $\widetilde{S}_{0}$ of the full preimage of $S_{0}$ in $\widetilde{S}$ and let $C$ be the intersection of the closure of $\widetilde{S}_{0}$ in $D$ with $S_{\infty}$. Then $C$ is a Cantor set if $S \neq S_{0}$ and $C=S_{\infty}$ if $S=S_{0}$. Since $C$ contains at least three points there is at most one lift of any homeomorphism that pointwise fixes $C$.

Given $h \in \mathcal{H}_{0}$ choose $g: S \rightarrow S$ that is isotopic to $h$ and pointwise fixes $S_{0}$ and let $\tilde{g}: \widetilde{S} \rightarrow \widetilde{S}$ be the lift of $g$ that pointwise fixes $\widetilde{S}_{0}$. The isotopy from $h$ to $g$ lifts to 
an isotopy from $\tilde{h}$ to a lift $\tilde{g}$ of $g$ satisfying $\left.\widehat{h}\right|_{C}=\left.\hat{g}\right|_{C}=$ identity. The assignment $h \rightarrow \widehat{h}$ defines a lift $\widehat{\mathcal{H}_{0}} \subset \operatorname{Homeo}(D)$ of $\mathcal{H}_{0}$.

Given $y \in \operatorname{Fix}\left(\alpha^{n}\right) \cap \operatorname{int}\left(S_{0}\right)$, choose a lift $\tilde{y} \in \widetilde{S}_{0}$ of $y$, let $A: \widetilde{S} \rightarrow \tilde{S}$ be the lift of $\alpha^{n}$ that fixes $\tilde{y}$ and note that $A$ preserves $\widetilde{S}_{0}$. Then $C$ is $\hat{A}$-invariant and $\operatorname{Fix}\left(\left.\widehat{A}\right|_{S_{\infty}}\right)$ is a finite subset of $C$ with more than two elements. Moreover, each point of Fix $\left(\left.\widehat{A}\right|_{S_{\infty}}\right)$ is either an attracting or repelling fixed point for $\widehat{A}: D \rightarrow D$ (see, for example, Theorem 5.5 of Casson and Bleiler [4] or Handel and Thurston [13]). The isotopy from $\alpha^{n}$ to $f^{n}$ lifts to an isotopy from $A$ to a lift $F$ of $f^{n}$ such that $\widehat{F}=\widehat{A}$. The commutator $[\widehat{F}, \widehat{h}]$ is the identity because it fixes each point in $C$ and is the extension of a lift of $\left[f^{n}, h\right]=$ identity. This proves that $\hat{F}$ commutes with each $\hat{h}$.

Theorem 1.1 applies to $\mathcal{G}=\mathcal{G}_{0}=\left\langle\widehat{\mathcal{H}_{0}}, F\right\rangle$. Thus there exists $\tilde{x} \in \operatorname{Fix}\left(\widehat{\mathcal{H}_{0}}\right) \cap \operatorname{Fix}(F) \cap \tilde{S}$. The image $x \in S$ of $\tilde{x}$ satisfies the conclusions of the theorem.

The following result is implicit in the proof of Lemma (3.3.5) of Calegari [3].

Proposition 3.1 . Let $G$ be a finitely generated group which admits a nontrivial $C^{1}$ action on a connected surface $S$ in such a way that $\operatorname{Fix}(G)$ contains a nonisolated point. Then there is a nontrivial homomorphism $\phi: G \rightarrow \mathbb{R}$, ie $H^{1}(G, \mathbb{R})$ is nontrivial.

Proof Choose a nonisolated point $x$ of $\operatorname{Fix}(G)$. The assignment $g \mapsto \operatorname{det}\left(D g_{x}\right)$ defines a homomorphism from $G$ to $\mathbb{R}$. If this is nontrivial we are done. Otherwise, each $D g_{x}$ has determinant one. Since $x$ is the limit of global fixed points there is a vector based at $x$ that is fixed by each $D g_{x}$. Thus there is a basis for the tangent space of $S$ at $x$ with respect to which each

$$
D g_{x}=\left(\begin{array}{cc}
1 & x_{g} \\
0 & 1
\end{array}\right)
$$

The map $g \mapsto x_{g}$ defines a homomorphism from $G$ to $\mathbb{R}$. If it is nontrivial we are done. Otherwise $x_{g}=0$ and $D g_{x}$ is the identity for all $g \in G$. The existence of a nontrivial homomorphism from $G$ to $\mathbb{R}$ now follows from the Thurston stability theorem ([20], see also Theorem 3.4 of [7]).

Proof of Theorem 1.6 Assuming there is a faithful homomorphism $\mathcal{L}: \operatorname{MCG}(S) \rightarrow$ $\operatorname{Diff}(S)$ satisfying (1) and (2), we prove that there is a nontrivial homomorphism from $\mathcal{L}\left(\Gamma_{1}\right)$ to $\mathbb{R}$, thereby contradicting the assumption that $H^{1}\left(\Gamma_{1}, \mathbb{R}\right)$ is trivial.

By hypothesis, each $h \in \mathcal{L}\left(\Gamma_{1}\right)$ is isotopic to a homeomorphism that is the identity on $S_{2}$ and $f:=\mathcal{L}(\mu)$ is isotopic to a homeomorphism $\alpha$ such that $\alpha\left(S_{2}\right)=S_{2}$ and $\left.\alpha\right|_{S_{2}}$ is pseudo-Anosov. Also $f$ commutes with each $h \in \mathcal{L}\left(\Gamma_{1}\right)$ because $\mu$ commutes 
with each element of $\Gamma_{1}$. Theorem 1.4 implies that $\operatorname{Fix}\left(\mathcal{L}\left(\Gamma_{1}\right)\right)$ is infinite and hence has an accumulation point. The existence of a nontrivial homomorphism from $\mathcal{L}\left(\Gamma_{1}\right)$ to $\mathbb{R}$, now follows from Proposition 3.1.

\section{References}

[1] C Bonatti, Un point fixe commun pour des difféomorphismes commutants de $S^{2}$, Ann. of Math. (2) 129 (1989) 61-69 MR979600

[2] C Bonatti, Difféomorphismes commutants des surfaces et stabilité des fibrations en tores, Topology 29 (1990) 101-126 MR1046627

[3] D Calegari, Circular groups, planar groups, and the Euler class, from: "Proceedings of the Casson Fest”, (C Gordon, Y Rieck, editors), Geom. Topol. Monogr. 7 (2004) 431-491 MR2172491

[4] A J Casson, S A Bleiler, Automorphisms of surfaces after Nielsen and Thurston, London Math. Soc. Student Texts 9, Cambridge University Press (1988) MR964685

[5] B Farb, Some problems on mapping class groups and moduli space, from: "Problems on mapping class groups and related topics”, Proc. Sympos. Pure Math. 74, Amer. Math. Soc. (2006) 11-55 MR2264130

[6] A Fathi, F Laudenbach, V Poenaru, et al, Travaux de Thurston sur les surfaces, Séminaire Orsay, Astérisque 66, Société Mathématique de France, Paris (1979) MR568308 French with an English summary

[7] J Franks, Distortion in groups of circle and surface diffeomorphisms, from: "Dynamique des difféomorphismes conservatifs des surfaces: un point de vue topologique", Panor. Synthèses 21, Soc. Math. France, Paris (2006) 35-52 MR2288284

[8] J Franks, M Handel, Distortion elements in group actions on surfaces, Duke Math. J. 131 (2006) 441-468 MR2219247

[9] J Franks, M Handel, K Parwani, Fixed points of abelian actions, J. Mod. Dyn. 1 (2007) 443-464 MR2318498

[10] J Franks, M Handel, K Parwani, Fixed points of abelian actions on $S^{2}$, Ergodic Theory Dynam. Systems 27 (2007) 1557-1581 MR2358978

[11] M Handel, Commuting homeomorphisms of $S^{2}$, Topology 31 (1992) 293-303 MR1167171

[12] M Handel, A fixed-point theorem for planar homeomorphisms, Topology 38 (1999) 235-264 MR1660349

[13] M Handel, W P Thurston, New proofs of some results of Nielsen, Adv. in Math. 56 (1985) 173-191 MR788938

[14] S P Kerckhoff, The Nielsen realization problem, Ann. of Math. (2) 117 (1983) 235-265 MR690845 
[15] M Korkmaz, Low-dimensional homology groups of mapping class groups: a survey, Turkish J. Math. 26 (2002) 101-114 MR1892804

[16] V Markovic, Realization of the mapping class group by homeomorphisms, Invent. Math. 168 (2007) 523-566 MR2299561

[17] J N Mather, Topological proofs of some purely topological consequences of Carathéodory's theory of prime ends, from: "Selected studies: physics-astrophysics, mathematics, history of science", North-Holland, Amsterdam (1982) 225-255 MR662863

[18] S Morita, Characteristic classes of surface bundles, Invent. Math. 90 (1987) 551-577 MR914849

[19] S Morita, Geometry of characteristic classes, Translations of Math. Monogr. 199, Amer. Math. Soc. (2001) MR1826571 Translated from the 1999 Japanese original, Iwanami Series in Modern Mathematics

[20] W P Thurston, A generalization of the Reeb stability theorem, Topology 13 (1974) 347-352 MR0356087

Department of Mathematics, Northwestern University

Evanston, IL 60208

Department of Mathematics, Lehman College

Bronx, NY 10468

john@math.northwestern.edu, michael.handel@lehman. cuny.edu

Proposed: Benson Farb

Seconded: Leonid Polterovich, Shigeyuki Morita
Received: 23 April 2008

Revised: 9 September 2008 